\title{
Treating Choroidal Metastases and Improving Vision with Osimertinib in EGFR T790M-Mutated Lung Adenocarcinoma: A Case Report and Review of the Literature
}

\author{
Karim Keshwani ${ }^{a}$ Kelsey A. Roelofs ${ }^{b}$ Gordon Hay ${ }^{c}$ Rachel Lewis $^{a}$ \\ Nick Plowman ${ }^{a}$ \\ a Barts Cancer Centre, St Bartholomew's Hospital, Barts Health NHS Trust, London, UK; ${ }^{\text {b } M o o r f i e l d s ~ E y e ~ H o s p i t a l ~}$ \\ Department of Ocular Oncology, London, UK; ${ }^{C}$ Moorfields Eye Hospital Department of Ocular Oncology, UCL \\ Institute of Ophthalmology and Barts Health NHS Trust, London, UK
}

\section{Established Facts}

- Lung cancer is the second most common primary tumour associated with uveal metastasis after breast cancer, accounting for roughly one-quarter of the cases.

- Several treatments have previously been used to treat uveal metastasis, including external beam radiotherapy, ruthenium or iodine plaque brachytherapy, photodynamic therapy, systemic chemotherapy, and more recently, systemic immunotherapy.

- Osimertinib is a third-generation epidermal growth factor receptor (EGFR) tyrosine kinase inhibitor (TKI) licensed for T790M mutation-positive lung adenocarcinoma.

\section{Novel Insights}

- Systemic treatment with osimertinib resulted in marked clinical regression of the left choroidal metastasis and a dramatic improvement in vision, even after prior TKI therapy, thereby negating the need for any additional local ocular treatment.

- As more small molecule-targeted therapies become available, a shift from focal to systemic treatments for choroidal metastases is likely to occur in the future. The dramatic response seen in this case highlights the value in carefully proceeding with close observation when a new systemic medication is introduced, even after prior systemic treatment failure, as the need for focal treatment may be spared.

\section{Keywords}

Choroidal metastases · Osimertinib · Tyrosine kinase inhibitors · Lung cancer

\begin{abstract}
A 41-year-old never-smoking female was diagnosed with epidermal growth factor receptor (EGFR)-mutated T2bN3M1b lung adenocarcinoma with axillary lymph nodes. She complained of blurred vision in the left eye $(2 / 60)$ and was subsequently found to have a left choroidal metastasis.
\end{abstract}


Treatment with tyrosine kinase inhibitor (TKI) erlotinib was started, and after 1 year of disease stability, she developed unsteadiness and worsening visual disturbance (1/60). Brain imaging showed 24 new brain metastases, which were treated with Gamma Knife stereotactic radiosurgery. An enlarging axillary lymph node was biopsied, which identified the T790M mutation, and she commenced the novel TKI osimertinib. Three weeks later, her choroidal lesion had regressed from $3.1 \mathrm{~mm}$ to $2.2 \mathrm{~mm}$, and after 2 months of osimertinib, her visual acuity had improved to 6/9. At the last follow-up 8 months after initiation of osimertinib, her choroidal metastasis remains stable, and visual acuity has improved to 6/6. Evidence suggests that osimertinib's efficacy in treating cerebral metastases is superior to that of chemotherapy and other EGFR-TKIs (gefitinib and erlotinib); however, the literature is sparse with regards to the use of osimertinib for the treatment of intraocular disease. In this case, the need for intense daily radiation treatment with its associated toxicities was negated, and as such we propose that osimertinib may be a promising treatment for choroidal metastasis secondary to EGFR-mutated lung adenocarcinoma.

(c) 2020 S. Karger AG, Basel

\section{Introduction}

The choroid is the most common (88\%) site of intraocular metastasis, owing to its rich vascular supply [1]. Several treatments for choroidal metastasis have been described, including external beam [2] or proton beam radiotherapy [3], photodynamic therapy [4], plaque brachytherapy [5], transpupillary thermotherapy [6], systemic chemotherapy [7], hormone therapy [8], and more recently, systemic immunotherapy.

Lung cancer represents the second most common cause of uveal metastasis (after breast cancer), accounting for roughly one-quarter (27\%) of the cases and typically presents with unilateral involvement [1]. In a series of 194 patients with uveal metastasis from lung cancer, Shah et al. [9] found that vision could be improved or stabilized in many patients (59\%); however, while treatment was effective at achieving local control in the majority (78\%), systemic prognosis was poor, with cancer-related death in 54\% 1 year following diagnosis with uveal metastasis. In the last 20 years, lung adenocarcinoma has replaced squamous cell carcinoma as the most prevalent lung nonsmall-cell lung cancer (NSCLC), with notable marked increased incidence in women.

Treating Choroidal Metastases with Osimertinib
More recently, epidermal growth factor receptor (EGFR) tyrosine kinase inhibitors (TKIs) have shown promise in treating patients with EGFR-mutated NSCLC with leptomeningeal metastasis [10]. A case of choroidal metastasis that showed a dramatic response to osimertinib, with improvement in both lesion size and vision, is presented herein.

\section{Case Report}

A 41-year-old previously fit, never-smoking female was diagnosed with metastatic EGFR-mutated lung adenocarcinoma (T2bN3M1b) in July 2017, with cervical, axillary, and pelvic lymph nodes. She also reported a few weeks of visual disturbance and blur in the left eye. Upon further evaluation, her visual acuity was $6 / 5$ in the right eye and 2/60 in the left. Dilated fundus examination revealed a creamy yellow lesion in the posterior pole, measuring $3.4 \mathrm{~mm}$ in elevation on ultrasound (Fig. 1). She was commenced on erlotinib in July 2017, a small-molecule TKI used first line in advanced lung adenocarcinoma with EGFR mutations.

Her choroidal metastasis responded well to erlotinib with an improvement in her vision (6/5 both eyes) by December 2017 . Her disease remained stable for several months until she developed unsteadiness and subtle worsening visual disturbance in April 2018; imaging revealed 24 new brain metastases. The brain metastases were treated with Gamma Knife stereotactic radiosurgery the same month. Her extracranial disease remained controlled, and she therefore continued on erlotinib. She had further Gamma Knife surgeries to two further cerebral lesions in November 2018 and did well until January 2019 when she noted a sudden decline in the vision in her left eye to $3 / 60$ and a worsening of her lymphadenopathy, with palpable axillary and inguinal lymph nodes. This indicated disease progression, and she underwent a lymph node biopsy to identify her T790M mutation status for second-line treatment options. She was concurrently referred for consideration of external beam radiotherapy (EBRT) to her choroidal metastasis; she declined to proceed with radiotherapy and therefore continued with observation of the choroidal metastasis while her biopsy result was awaited. The lymph node biopsy revealed a T790M mutation, and the patient was started on osimertinib in June 2019, 1 month after stopping erlotinib.

By this time, she had continued progression of her ocular disease, with significant worsening of the exudative retinal detachment. Three weeks after starting osimertinib, in July 2019, she felt that the visual field of her left eye was improving. Her visual acuity at this time was still rather poor (6/60). By August 2019 , her visual acuity had recovered to 6/9. At the most recent follow-up in February 2020, her visual acuity has improved to $6 / 6$, and the thickness of her choroidal metastasis has decreased to $1.7 \mathrm{~mm}$.

With respect to systemic side effects from osimertinib, she had acneform rashes and skin dryness affecting her activities of daily living, but this has been controlled with oral antibiotics, topical ointments, and a $50 \%$ dose reduction. 
Fig. 1. a Fundoscopic examination revealed a pale lesion in the inferior macula of the left eye. b Optical coherence tomography (OCT) confirmed the choroidal location of this lesion and demonstrated shallow subretinal fluid. Following treatment with erlotinib, her vision (6/5 both eyes) by December 2017 and fundoscopic examination and OCT (c, d) showed significant regression of the metastatic lesion. She remained stable until January 2019 when her choroidal metastasis increased in both basal (e) and apical (f) dimensions and re-accumulated subretinal fluid, accompanied by a decrease in her vision $(3 / 60)$. g, h Her choroidal metastasis continued to progress, with a significant increase in the amount of subretinal exudates and fluid by July 2019. i, j) Following 3 weeks of treatment with osimertinib, there was a marked regression of the choroidal metastasis with significant reduction in the associated exudative retinal detachment. k, I Her fundus appearance and visual acuity $(6 / 6)$ has remained stable through to her most recent follow-up, 8 months following treatment with osimertinib.
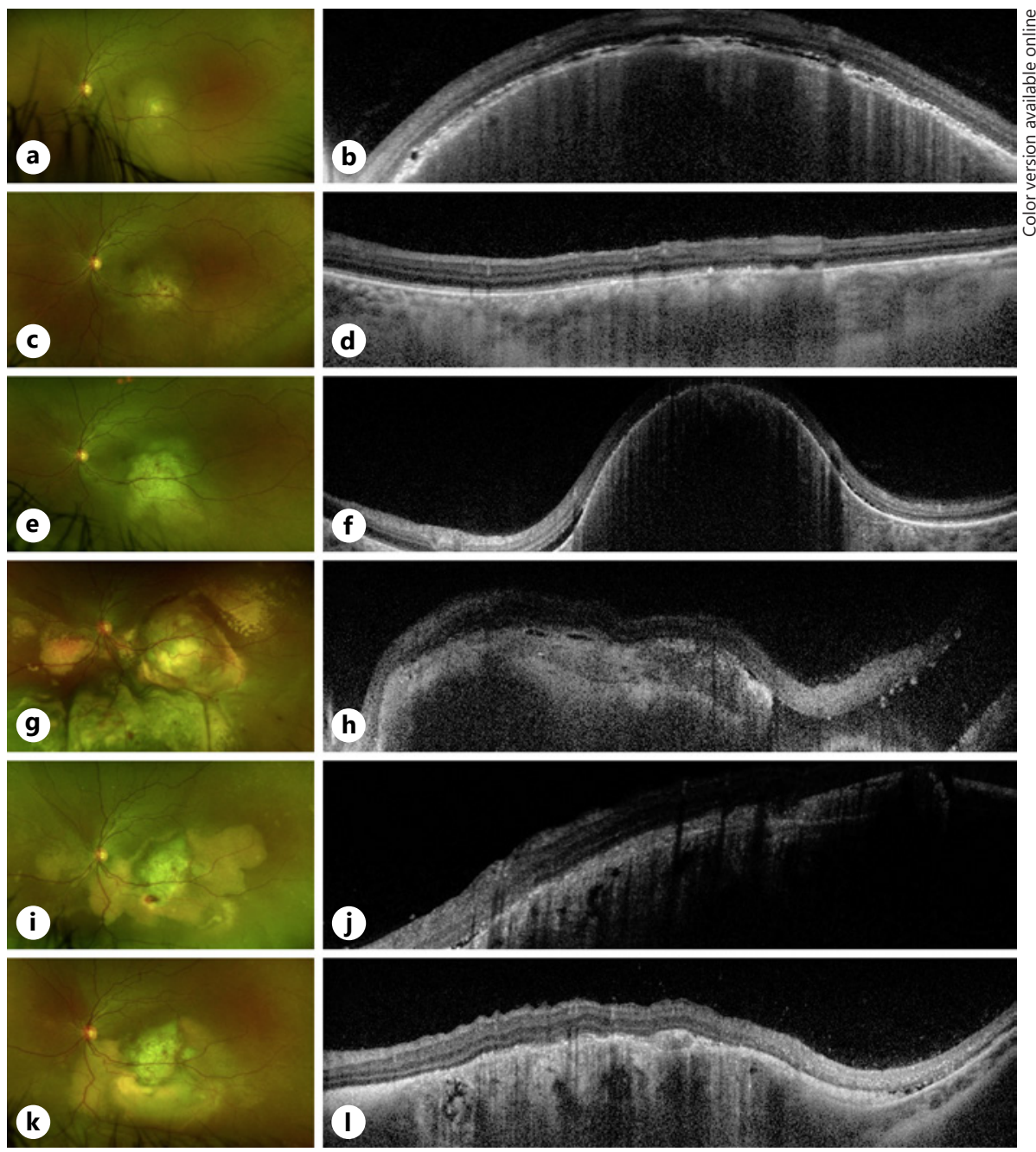

\section{Discussion}

The promising results seen in the BLOOM study [10] support previous reports suggesting that osimertinib effectively penetrates the central nervous system (CNS). As there are many similarities in the privilege afforded by both the blood-brain and blood-ocular barrier, it is intuitive that a medication with good CNS penetration may also treat intraocular disease more effectively.

In a review of 194 patients with uveal metastasis from lung cancer, Shah et al. [9] reported that $30 \%$ of the patients were treated with EBRT and an additional 22\% received a combination of EBRT and systemic chemotherapy with the majority of eyes (74\% with EBRT alone; $82 \%$ with EBRT + systemic chemotherapy) showing regression. Overall, $59 \%$ of the patients included in this study had stabilization or improvement of vision. However, treatment with radiotherapy has several potential drawbacks, including the burden of multiple treatment visits when administered in fractionated doses, and ocular complications including cataract (7\%), exposure keratopathy (3\%), or radiation retinopathy (3\%) [2].

In patients with multiple foci of metastases, systemic treatment has several obvious advantages: a once-daily oral preparation, ability to treat various sites of disease, reduced burden of frequent hospital visits, and avoidance of the toxicities associated with local treatments such as radiotherapy.

To the best of our knowledge, there have been only two prior reports documenting response of choroidal metastasis to systemic treatment with osimertinib $[11,12]$. Similar to our case, both of these patients were initially treated with erlotinib, but eventual disease progression necessitated the switch to osimertinib as second-line treatment. 
Nair et al. [13] reported a case of choroidal metastasis from NSCLC successfully treated with erlotinib. In this review, the authors summarized 8 other cases [14-19] of choroidal metastasis from NSCLC treated with first-generation EGFR-TKIs in the literature (erlotinib and gefitinib), finding that the majority of cases improved following treatment with TKIs, thereby suggesting that this class of medication may play an important role in the management of this cohort of patients.

Osimertinib is a third-generation EGFR-TKI, irreversibly inhibiting EGFR, a transmembrane glycoprotein receptor. It is indicated as monotherapy in the second-line setting for the treatment of locally advanced or metastatic EGFR T790M mutation-positive NSCLC. Over $50 \%$ of the acquired resistance to EGFR-TKIs involves this mutation, where there is substitution of a threonine residue for the larger methionine at position 790 .

The United States Food and Drug Administration (FDA) and the European Medicines Agency (EMA) have approved the use of first-line osimertinib for metastatic NSCLC tumours with EGFR exon 19 deletion or exon 21 L858R mutation. The National Institute for Health and Care Excellence (NICE) recommended erlotinib, gefitinib, afatanib and most recently, dacomitinib as first-line treatment in EGFR-mutated NSCLC, with osimertinib recommended as a second-line agent only, following circulating tumour cell or biopsy-proven T790M mutation [20].

This decision was particularly contentious given recently matured evidence that first-line osimertinib improves overall survival in untreated EGFR-mutated advanced lung cancer (38.6 months median overall survival) versus two comparator EGFR-TKIs, erlotinib and gefitinib (31.8 months HR 0.799 [ $p=0.0462]$ ), with more than half of the patients (54\%) alive in the osimertinib group compared to $44 \%$ in the comparator group at 3 years [21].

Although the study was not powered to demonstrate statistical significance in subgroup analyses, results were interesting with regard to CNS disease: a planned progression-free survival (PFS) subgroup analysis reported at 18 months was $58 \%$ in the osimertinib group and $40 \%$ in the comparator group (HR 0.48, CI 0.26 to 0.86 ) [22]. There was also reduced CNS progression in those without or with treated CNS disease in the osimertinib arm ( 7 out of 226 [3\%]) compared to the comparison drug arm (15 out of 214 [7\%]) [23]. While there are many potential explanations for this, some have hypothesized that osimertinib may prevent the development of brain or ocular metastases. It is also worth noting that intra- and extracranial responses to osimertinib were concordant by $77 \%$

Treating Choroidal Metastases with

Osimertinib
[24]. The high concordance reported between both intraand extra-cranial response to osimertinib supports its ability to effectively cross the blood-brain and perhaps, blood-ocular barrier.

Other EGFR-targeted TKIs have also demonstrated improvements in intracranial PFS: Afatinib is a secondgeneration EGFR-TKI used successfully as first-line therapy to improve PFS in patients with asymptomatic brain metastases compared to chemotherapy [25]. PFS was also improved with afatanib in pre-treated patients with prior chemotherapy or other EGFR-TKIs, with a $66 \%$ cerebral disease control rate [26]. No trial has directly compared efficacy of afatanib and osimertinib to date.

During the COVID-19 pandemic, osimertinib was subsequently made available first line by NHS England to mitigate the risk of contracting the virus; oral administration in a setting that reduces exposure to COVID-19 such as at home, as well as being less resource-intensive and immunosuppressive than chemotherapy, were factors considered in this change, which is expected to remain in place in future, allowing treatment-naïve patients to continue to receive osimertinib first line.

\section{Conclusion}

Although several established TKIs including erlotinib and gefitinib, are often used as first-line treatment in patients with EGFR-mutated lung adenocarcinoma, this case highlights the potential of osimertinib in treating concurrent uveal metastasis. Although osimertinib is currently indicated as second-line treatment in the UK, there is growing evidence in support of its ability to improve overall and PFS, in particular for intracranial and perhaps intraocular disease. When initiating treatment with osimertinib in patients with uveal metastasis, it is prudent to pause for a short period of observation prior to proceeding with EBRT in the event that systemic TKI in and of itself is sufficient to treat the intraocular disease, even in the presence of prior TKI intraocular disease failure. Osimertinib is anticipated to become more commonplace in the treatment of cerebral and ocular disease and therefore worthwhile becoming acquainted with.

\section{Statement of Ethics}

This research was conducted in accordance with the Declaration of Helsinki. The subject has given written informed consent to publish this case. No identifying information or photographs are published in this study. 


\section{Conflict of Interest Statement}

None of the authors have any financial disclosures or conflicts of interest to declare.

\section{Author Contributions}

All authors have contributed to the writing and approval of the final version of the manuscript.

\section{Funding Sources}

No funding was obtained for this study.

\section{References}

1 Shields CL, Shields JA, Gross NE, Schwartz GP, Lally SE. Survey of 520 eyes with uveal metastases. Ophthalmology. 1997 Aug; 104(8):1265-76.

2 Rudoler SB, Corn BW, Shields CL, De Potter P, Hyslop T, Shields JA, et al. External beam irradiation for choroid metastases: identification of factors predisposing to long-term sequelae. Int J Radiat Oncol Biol Phys. 1997 May;38(2):251-6.

3 Tsina EK, Lane AM, Zacks DN, Munzenrider JE, Collier JM, Gragoudas ES. Treatment of metastatic tumors of the choroid with proton beam irradiation. Ophthalmology. 2005 Feb; 112(2):337-43.

4 Ghodasra DH, Demirci H. Photodynamic therapy for choroidal metastasis. Am J Ophthalmol. 2016 Jan;161:104-9.e1.

5 Shields CL, Shields JA, De Potter P, Quaranta M, Freire J, Brady LW, et al. Plaque radiotherapy for the management of uveal metastasis. Arch Ophthalmol. 1997 Feb;115(2):203-9.

6 Romanowska-Dixon B, Kowal J, Pogrzebielski A, Markiewicz A. [Transpupillary thermotherapy (TTT) for intraocular metastases in choroid]. Klin Oczna. 2011;113(4-6):132-5.

7 Kosmas C, Malamos NA, Antonopoulos M. Complete regression of choroidal metastases from breast cancer after docetaxel-based systemic chemotherapy. Med Pediatr Oncol. 2000 Mar;34(3):229-30.

8 Manquez ME, Brown MM, Shields CL, Shields JA. Management of choroidal metastases from breast carcinomas using aromatase inhibitors. Curr Opin Ophthalmol. 2006 Jun; 17(3):251-6.

9 Shah SU, Mashayekhi A, Shields CL, Walia HS, Hubbard GB 3rd, Zhang J, et al. Uveal metastasis from lung cancer: clinical features, treatment, and outcome in 194 patients. Ophthalmology. 2014 Jan;121(1):352-7.

10 Yang JC, Kim SW, Kim DW, Lee JS, Cho BC Ahn JS, et al. Osimertinib in Patients With Epidermal Growth Factor Receptor Mutation-Positive Non-Small-Cell Lung Cancer and Leptomeningeal Metastases: the BLOOM Study. J Clin Oncol. 2020 Feb;38(6):538-47.
11 Dall'Olio FG, Ruatta C, Melotti B, Sperandi F, Ciardella AP, Ardizzoni A. Response to Osimertinib in Choroidal Metastases from EGFRmt T790M-Positive Non-Small Cell Lung Adenocarcinoma. J Thorac Oncol. 2017 Oct;12(10):e165-7.

12 Mariachiara M, Celeste R, Federico F, Nicole B, Antonio C. Choroidal metastasis from non-small-cell lung cancer responsive to Osimertinib: a case report : Efficacy of a thirdgeneration epidermal growth factor tyrosine kinase inhibitor. Int Ophthalmol. 2018 Dec; 38(6):2669-75.

13 Nair AG, Asnani HT, Mehta VC, Mehta SV, Pathak RS, Palkar AH, et al. Tyrosine Kinase Inhibitors in the Treatment of Choroidal Metastases from Non-Small-Cell Lung Cancer: A Case Report and Review of Literature. Ocul Oncol Pathol. 2017 Jan;3(1):28-33.

14 Ye X, Kaliki S, Shields CL. Rapid regression of choroidal metastasis from lung cancer using erlotinib (Tarceva). Oman J Ophthalmol. 2014 May;7(2):75-7.

15 Daniels AB, Miller ML, Kotecha A, Abramson $\mathrm{DH}$. Uveal metastasis from nonsmall cell lung carcinoma with dramatic response to erlotinib. Retin Cases Brief Rep. 2010;4(4):390-3.

16 Kim SW, Kim MJ, Huh K, Oh J. Complete regression of choroidal metastasis secondary to non-small-cell lung cancer with intravitreal bevacizumab and oral erlotinib combination therapy. Ophthalmologica. 2009;223(6): 411-3.

17 Fujiu K, Kobayashi N, Miyamoto H, Suzuki $\mathrm{H}$. A case of choroidal metastasis of lung cancer successfully treated with erlotinib. Gan To Kagaku Ryoho. 2012 Feb;39(2):269-71.

18 Hirsh V. Second-line erlotinib in an EGFR mutation-negative patient with non-smallcell lung cancer. Curr Oncol. 2012 Feb;19(1): 21-2.

19 Singh N, Kulkarni P, Aggarwal AN, Rai Mittal B, Gupta N, Behera D, et al. Choroidal metastasis as a presenting manifestation of lung cancer: a report of 3 cases and systematic review of the literature. Medicine (Baltimore). 2012 Jul;91(4):179-94.
20 Lung cancer: diagnosis and management. NICE Guidance [Internet]. Nice.org.uk. 2020 [cited 3 June 2020]. Available from: https:// www.nice.org.uk/guidance/ng122.

21 Ramalingam S, Gray J, Ohe Y, Cho B, Vansteenkiste J, Zhou C, et al. Osimertinib vs comparator EGFR-TKI as first-line treatment for EGFRm advanced NSCLC (FLAURA): final overall survival analysis. Ann Oncol. 2019; 30:v914-5.

22 Ramalingam SS, Vansteenkiste J, Planchard D, Cho BC, Gray JE, Ohe Y, et al.; FLAURA Investigators. Overall Survival with Osimertinib in Untreated, EGFR-Mutated Advanced NSCLC. N Engl J Med. 2020 Jan;382(1):4150.

23 Soria JC, Ohe Y, Vansteenkiste J, Reungwetwattana $\mathrm{T}$, Chewaskulyong $\mathrm{B}$, Lee $\mathrm{KH}$, et al. FLAURA Investigators. Osimertinib in Untreated EGFR-Mutated Advanced NonSmall-Cell Lung Cancer. N Engl J Med. 2018 Jan;378(2):113-25.

24 Reungwetwattana T, Nakagawa K, Cho BC, Cobo M, Cho EK, Bertolini A, et al. CNS Response to Osimertinib Versus Standard Epidermal Growth Factor Receptor Tyrosine Kinase Inhibitors in Patients With Untreated EGFR-Mutated Advanced Non-Small-Cell Lung Cancer [published online ahead of print, 2018 Aug 28]. J Clin Oncol. 2018 Aug; CO2018783118(33):JCO2018783118.

25 Schuler M, Wu YL, Hirsh V, O’Byrne K, Yamamoto N, Mok T, et al. First-Line Afatinib versus Chemotherapy in Patients with NonSmall Cell Lung Cancer and Common Epidermal Growth Factor Receptor Gene Mutations and Brain Metastases. J Thorac Oncol. 2016 Mar;11(3):380-90.

26 Hoffknecht P, Tufman A, Wehler T, Pelzer T, Wiewrodt R, Schütz M, et al.; Afatinib Compassionate Use Consortium (ACUC). Efficacy of the irreversible ErbB family blocker afatinib in epidermal growth factor receptor (EGFR) tyrosine kinase inhibitor (TKI)-pretreated non-small-cell lung cancer patients with brain metastases or leptomeningeal disease. J Thorac Oncol. 2015 Jan;10(1):156-63. 